\title{
Contribution of Risk Perception for the Analysis of Non-Compliance to Work Procedures
}

\author{
Mohammed Mouda1, Mébarek Djebabra', Makhlouf Chati2 \\ ${ }^{1}$ LRPI Laboratory of Institute of Health and Safety, University of Batna, Batna, Algeria \\ ${ }^{2}$ HSE Department of Regional Direction of Hassi R'mel, Group of Sonatrach, Laghouat, Algeria \\ Email: mmouda@yahoo.fr, djebabra mebarek@yahoo.fr, chati dz@yahoo.fr
}

Received 17 September 2014; revised 31 October 2014; accepted 16 November 2014

Copyright (C) 2014 by authors and Scientific Research Publishing Inc.

This work is licensed under the Creative Commons Attribution International License (CC BY). http://creativecommons.org/licenses/by/4.0/

c) (i) Open Access

\begin{abstract}
One of the problems encountered in work security is non-compliance to work procedures by operators. The consequences of such human behavior are harmful to both the operators and the organization. These consequences are the result of a situational behavior of operators. This article focuses on the perception of work situations in a sample of Algerian workers in oil companies knowing that this perception determines, in large part, the decisions taken by the operators in terms of non-compliance with work procedures.
\end{abstract}

\section{Keywords}

Work Procedure, Non-Compliance, Human Behavior, Human Perception

\section{Introduction}

The study of accidents reveals that human error is the main cause of these accidents [1]. These errors are framed by operators who attempt to adapt working conditions which are not optimal and that are caused by the economic and organizational pressures as well as technical constraints [2].

To solve this problem, the automation of industrial processes is the first solution that comes to mind and that reduces human involvement at work. This solution has the disadvantage of creating new forms of error such as [3]: the phenomenon of loss of situational awareness, confusion between automatic and manual modes in some partially automated processes. Moreover, automation is problematic in developing countries because of problems of technology transfer [4].

Consequently, a second solution consists in controlling human behavior through the multiplication of proce- 
dures, regulations and standards [5]. This solution, practiced in many socio-technical systems with high risks, creates another problem in companies namely the non-compliance to Work Procedures (WP) by operators [6].

Indeed, the desire to achieve some objectives (production and deadline) is responsible for these violations of WP [7]. Obviously, more one procedure of work is overloaded in actions to be respected; more one has violations of these procedures [8]. In addition, the work procedure is as a list of actions to perform or not to perform, without justifications and without any conceptual models. A feeling of stress and loss of direction results from this. Especially the rules to be respected are sometimes useless according to operators.

In other words, the Non-Compliance with a WP (NC-WP) is initiated by behavioral excesses that reflect differences between what is really done and what should have been done. These excesses are associated with situational excesses which correspond to differences between the really obtained situation and that should have been obtained [9] [10].

In terms of scientific research, studies on NC-WP are focused essentially on situational deviations that are the result of behavioral ones [11]. Specifically, the analysis of deviations in terms of situational consequences associated with NC-WP (Figure 1).

In Figure 1, human behavior in terms of NC-WP is intentional. It is characterized by three main steps:

- The reflection step where the operator enters a reflection of NC-WP. This thinking is fueled by a desire to simplify the WP in order to lighten the workload.

- The action step where the operator realizes his desire for a NC-WP. This operator's action is initiated by criteria related primarily to: safety at work, productivity and workload.

Obviously, the operator assigns weights to these criteria and it is on the basis of their assessment that the operator undertakes (or not) his action. However, it is important to remember that these weights estimated by the operator are subjected to uncertainties that can make his action biased.

- The step linked to the immediate consequences arising from the action of the operator. These consequences can be harmful to the operator.

This guidance focused on intentional behavior must be, logically, preceded by the study of the perception of the risk at work, which is an important component to understand since it is situated in the upstream of the preventive process and is, thus considered to be a determining factor in the attitude and the human behavior when facing a risk at work [12]. It is in this context that this study is integrated which is focused on the perception of risks at work by operators in an Algerian oil field.

\section{Materials and Methods}

The proposed method is framed by a cognitive process of data processing and decision-making of NC-WP (Figure 2).

In Figure 2, the perception of the working situation is a complex process through which an operator: receives, processes and measures the information of his physical and communicative environment through the senses [13].

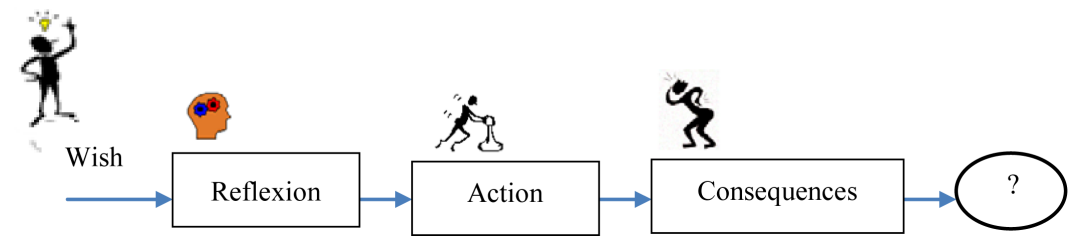

Figure 1. Intentional behavior of an operator to violate a WP.

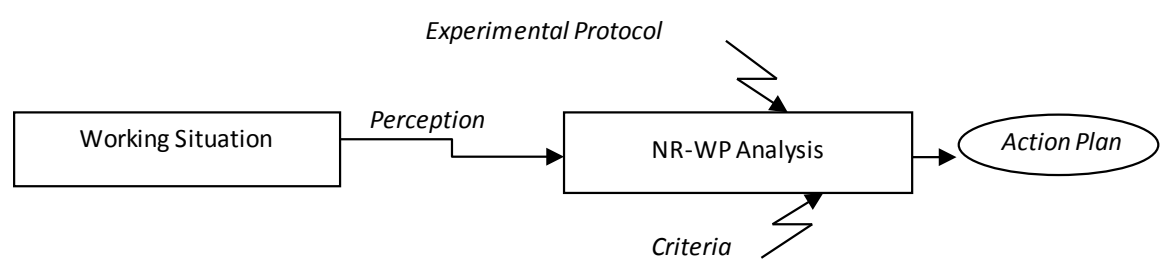

Figure 2. General framework of the proposed method. 
The experimentation is the best way to understand this perception [14] [15].

\subsection{The Experimental Protocol}

The loss of control is the framework of the protocol; because it is the most frequent cause of accidents at work due to the NP-WP in the Algerian oil fields [6]. This cause concerns: objects worn or handled, equipment (tools) and transportation and routing means.

In this context, the experimental protocol aims at identifying the operators' preferences for the NR-WP concerning the operations of pipes blowing in the studied oil field.

This experimental protocol is, therefore, a questionnaire whose structure is composed of three main parts:

- Identity: this section contains general information about the operators (age, seniority, training level, marital status, etc.).

- The attitudes towards risks at work (RW) where the focus is on:

o Aversion of RW (A-RT)

o Preference to the Present (PP)

o Working Conditions (WC) focused on: Musculoskeletal Disorders (MSDs) and the agreement of the protection measures.

- Opinions that embody the perception of the following criteria: Safety at work (S), productivity (P) and workload (W).

All those dimensions are measured by a score on three levels: $1=$ low, $2=$ moderate and $3=$ strong.

The advantage of this structure of the questionnaire is in its progressive character: we are first interested in the identity of the interviewees in order to better prepare them for the investigation and then to the information (behavior) which is "easier" to obtain to move progressively towards the more involving elements (opinions or preferences).

\subsection{Field and Timeline for Implementing the Method}

This study was conducted in an Algerian oil field. The target population for the study consisted of 48 operators whose average age was 43.5 years (age 21 to 61 years). The average seniority of this target population is 16.5 years (seniority 01 to 30 years). The target population level of education is the primary school level for half of the workforce studied (primary level $=48.9 \%$; mean level $=42.2 \%$ and university level $=8.9 \%$ ). $11.6 \%$ of the studied participants benefited from sick leave for causes of accidents at work.

The timing of the survey was spread over the whole year 2013. Along this period, our field observations showed that the work procedures relating to operations of the pipes blowing were not respected in the majority of cases; in particular the design of workplaces and means of communication between operators.

To illustrate this, we first remind that the blowing operation, which comes before the hydrostatic testing, is performed using a foam pig driven by compressed air. It helps to clean the pipe from all foreign matters that could have been introduced during its realization.

The equipments used in this operation are mentioned in Figure 3. Each operation of a pipe blowing is performed by two operators assigned as follows: an operator is responsible for injecting compressed air from the compressor into the pipe through the blowing head and a second operator is positioned at the other end of the

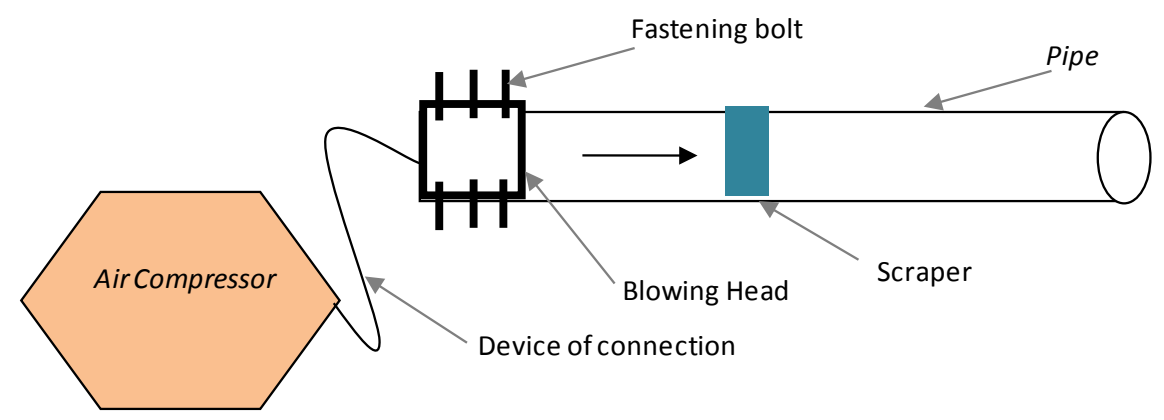

Figure 3. Operation of a pipe blowing. 
pipe to ensure the output of the scraper.

The two operators are in contact by means of communication until the end of the operation. Consequently, the main anomalies lie in: the design of workplaces that can corrupt communications between operators (presence of truck carrying the air compressor and strangers to the scene of operations (truck drivers).

To diagnose these anomalies, we mobilize the questionnaire information on the situational behavior of operators about the NC-WP.

\section{Results and Discussions}

As mentioned in the previous section, the developed questionnaire is composed of three parts: identities, behaviors and opinions of operators. Each of these three parts is framed by questions of unique closed-type (single modality to choose from a list of answers).

Our choice of this type of question is initiated by the simplicity of these questions, on the one hand, and our desire to avoid non-response operators, on the other. Despite this choice of closed questions, we had no-response to some questions (Table 1 ).

Note that the proposed answers to all the questions are limited in number (for 2 to 4 responses) which significantly reduced the no-responses to questions. Furthermore, the questions that have recorded a maximum rate of no-responses are those that characterize the social aspects of the operators. The explanation of these no-responses is related to the Algerian context and its political, economic and social changes since the last five decades. This context largely influences the working conditions of the operators.

The social aspects that have marked no-responses in some operators are, therefore, of great importance for our study. Because, they can track down what has to do with the human and social capital of the operator and are potential determinants of how to approach a risky situation. Consequently, the first part of our questionnaire survey elucidates not only the constituent factors of the social climate of the operators studied but also their importance to the operator's well-being when performing their professional activities. So:

- The education university level of some operators retained in this study is likely to be the cause of their behavior and opinions that differ from other categories of operators with limited levels of education at the elementary or secondary level. For example, for the A-RW dimension, operators of limited level of education seek to earn a living through this activity to preserve the random situations that the future holds without many concerns about the risks. This is not the case for university operators who intend to change this activity as soon as the opportunity arises. In other words, these university operators have a preference for the future while others have a preference for the present (Figure 4). Confirmation of this trend among university operators is validated by the statistical test Chi-square $(\mathrm{P}=0.74)$.

- Age is a priori a natural determinant of risk perception among the studied operators. This is the case of the agreement with the operator's effectiveness of prevention and protection that is proportionate to the operators' age. Figure 5 shows that actually the youngest have a low preference to these measures compared with the wisdom and experience of the oldest operators.

In Figure 5, we crosschecked the question of the operators age with the questions related to prevention and protection means (SAP, EAT, GEF, MPI and MPC). This is a cross-tabulation. The statistical test of chi-square is clearly validated: $\mathrm{P}=0.96$.

The second part of the questionnaire is devoted mainly to three dimensions of the RW perception, which are:

- A-RW where the results of this perception are provided in Figure 4.

- Preference to the present where all the interviewed operators have the preference of no longer thinking about the consequences associated to the risk of control loss. For them, this activity is certainly one of high risk and accidents are fatal. Therefore, operators attach great importance to the achievement of their business without considering the possible consequences.

Table 1. Distribution of non-operator responses to questions.

\begin{tabular}{cc}
\hline Questions & Percentage of no-responses to the questions \\
\hline 3, 7, 8 and 10 & 6.25 \\
5 & 4.17 \\
4 & 2.08 \\
\hline
\end{tabular}



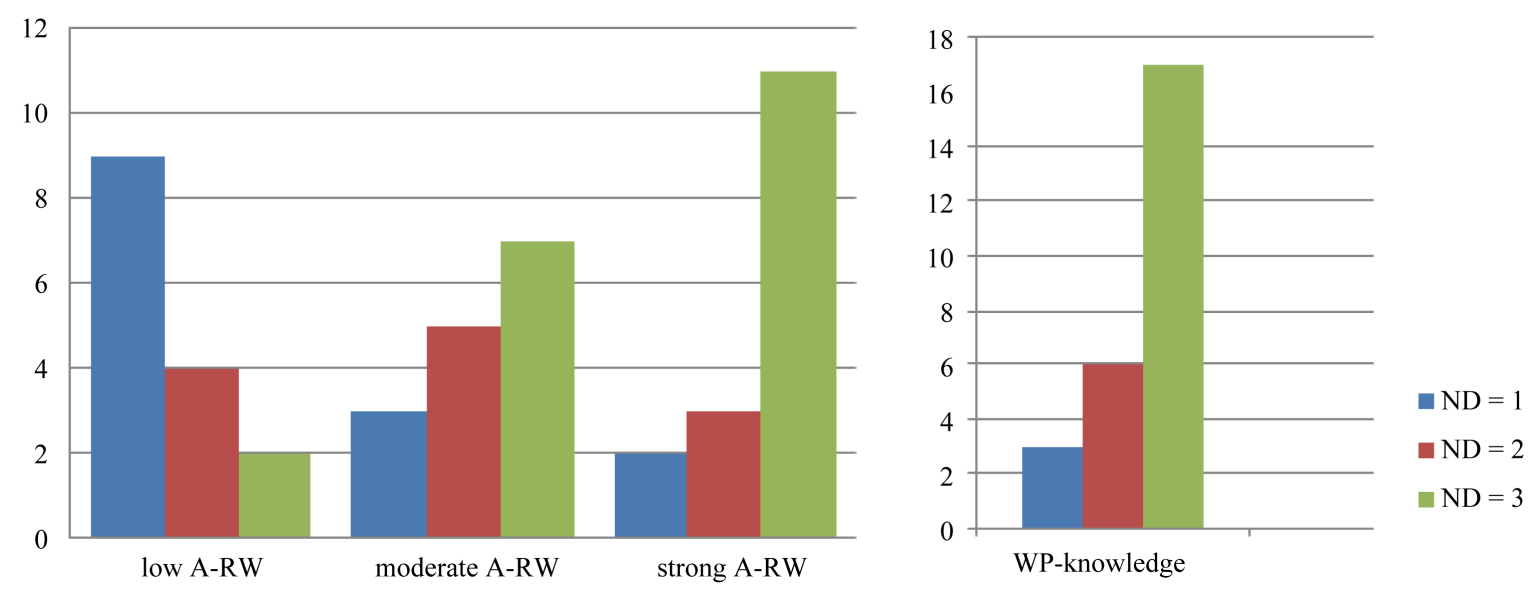

Figure 4. Opinions of operators on the A-RW dimension and on their knowledge of WP based on their levels of educations.

\begin{tabular}{l} 
MPC \\
\cline { 2 - 6 } \\
\cline { 2 - 6 }
\end{tabular}

- Working conditions for operators where the focus is on the protection measures of WR. In this context, the operators were asked about three key issues: the effectiveness of individual measures, the effectiveness of collective measures and the existence of critical periods of pipes blowing tasks.

Concerning the individual and collective measures of protection, the effectiveness of these measures is closely linked to their respect which is problematic for many reasons including those related to the lack of comfort when wearing individual measures and the loss of time when installing the equipment of blowing (Figure 5). Table 2 summarizes the results of these assessments.

As for the existence of critical periods in the pipes blowing, the majority of operators confirm it saying that it can either be in the beginning of work, where criticality is mainly due to problems with the preparation of the blowing equipment; or to the end of work where the criticality is materialized by operator fatigue and/or to problems of disassembly of the blowing equipment.

The third and final step of this investigation begins with information about the WR where $87 \%$ of operators say they were informed about the WR. This led us to ask them to assess the main causes of WR. The results of this assessment are provided in Figure 6, where we found that 92\% of these cases correspond to the NC-WP.

In order to find the correlation between the information on the WR and WR perception by operators used in this study, we investigated the NC-WP under criteria (S, P and W). In this context, we note that the preference for production (83\%) exceeds the one on safety at work (56\%) knowing that the workload is assessed to its maximum $86 \%$ by the operators. These results confirm the following hypothesis: the operators are well informed about the WR but this information is not well stored because production is given priority over safety. In 
Table 2. Extracts from the results of the investigation.

\begin{tabular}{|c|c|c|c|}
\hline \multicolumn{4}{|l|}{ Second part of questionnaire: the behaviors of the questioned operators } \\
\hline \multirow{2}{*}{ Dimensions and targeted parameters of the investigation } & \multicolumn{3}{|c|}{ Scores (in \%) } \\
\hline & Low & Moderate & Strong \\
\hline \multicolumn{4}{|l|}{ 1. Working conditions: } \\
\hline i. Painfulness of work & 4 & 13 & 83 \\
\hline \multicolumn{4}{|l|}{ ii. Effectiveness of prevention measurements } \\
\hline ii-1. Sensitizing with the problems involved in the physical activities & 10 & 48 & 42 \\
\hline ii-2. Heating before working & 15 & 60 & 25 \\
\hline ii-3. Training with the use of the material & 17 & 67 & 17 \\
\hline \multicolumn{4}{|l|}{ 2. Working conditions (agreement with the protection measures of the WR): } \\
\hline i. Effectiveness of the individual protection measures & 0 & 52 & 48 \\
\hline ii. Effectiveness of the collective protection measures & 0 & 40 & 60 \\
\hline \multicolumn{4}{|l|}{ Third part of questionnaire: the opinions of the questioned operators } \\
\hline 1. Information on the WR & 0 & 12 & 87 \\
\hline 2. Preference withe safety at work (S) & 4 & 40 & 56 \\
\hline 3. Preference with the production (P) & 4 & 13 & 83 \\
\hline 4. Workload or Painfulness of work (W) & 0 & 14 & 86 \\
\hline 5. Knowledge of WP & 19 & 33 & 48 \\
\hline 6. Degree of the WP respect & 26 & 74 & 0 \\
\hline
\end{tabular}

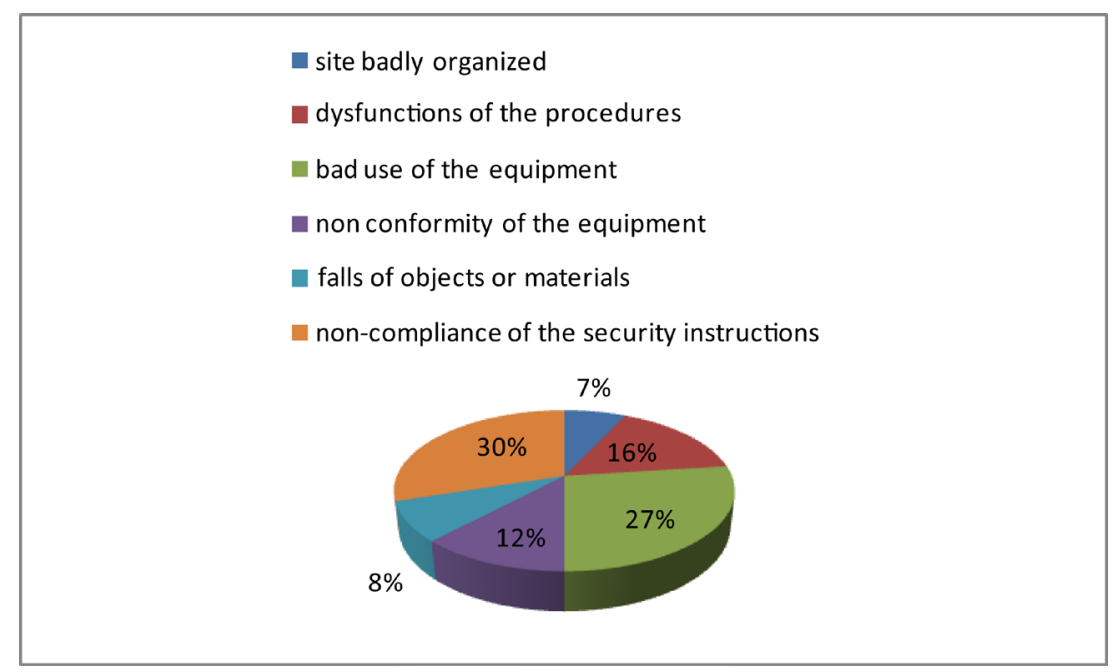

Figure 6. Causes of WR according to the studied builders.

this connection, we recall that over $54 \%$ of operators are young and display a low to average agreement to the prevention and protection measures (Figure 5).

Similarly, only $48 \%$ of the operators surveyed reported having knowledge of working procedures. Among this category of operators, $74 \%$ reported partial compliance with these procedures while the rest (26\%) affirm their NC-WP (Table 2).

The other results provided by these operators on the WP are: the activities period varies between 2 hours (19\%) and 3 hours (56\%), the average deviation from work for three hours is 30 minutes, the number of operations that make up an activity of pipes blowing ranges from: 5 (4\%), 6 (48\%) 7 (35\%) and 8 (13\%).

\section{Conclusions}

This article has demonstrated the value of the statistical study of the perception of working situations by operators in an Algerian oil field. At the end of this study, it appears that in order to improve WP and ensure their respects by the operators responsible for their implementation, it is important to consider the operators' skills, their capacity for autonomy as well as the working conditions that affect their behavior.

Moreover, the involvement of operators in the updating of WP wills, without a doubt ends this recurring 
problem of NC-WP. In this context, it is particularly important to capitalize this knowledge that is the basis for objectively measuring the performance of a WP.

\section{References}

[1] Stromme, M. (2013) Safety Training for the Oil and Gas Worker. J. J. Keller \& Associates, Inc.

[2] Leplat, J. and Rasmussen, J. (1984) Analysis of Human Errors in Industrial Incidents and Accidents for Improvement of Work Safety. Accident Analysis \& Prevention, 16, 77-88. http://dx.doi.org/10.1016/0001-4575(84)90033-2

[3] INRS Report (2012) Safety of the Machines: Operating Modes and Neutralized Protections. INRS Editions ID 6129, France.

[4] Rosenberg, N. and Frischtak, C. (1985) International Technology Transfer: Concepts, Measures and Comparisons. Praeger Editors, New York.

[5] Dudognon, K. and Soulard, N. (2004) Design and Setting in Conformity of the Machines and Working Equipments. Document of the Faculty of Pharmacy, Mediterranean University, Marseille.

[6] Mouda, M. and Djebabra, M. (2013) Proposition of a Participative Approach for the Information Risk Control: Application to Industrial Procedures Improvement. AWER Procedia Information Technology \& Computer Science, 3, 10041009.

[7] De Brito, Y. (2006) Statut et non-respect des procédures écrites. Intellectica, 44, 63-85.

[8] Hollnagel, E. (1993) The Phenotype of Erroneous Actions. International Journal of Man-Machine Studies, 39, 1-32. http://dx.doi.org/10.1006/imms.1993.1051

[9] Kohonen, T. (2001) Self-Organizing Maps. 3rd Edition, Springer, New York. http://dx.doi.org/10.1007/978-3-642-56927-2

[10] Lievens, F., Peeters, H. and Schollaert, E. (2008) Situational Judgment Tests: A Review of Recent Research. Personnel Review, 37, 426-441. http://dx.doi.org/10.1108/00483480810877598

[11] Analoui, F. (2007) Strategic Human Resource Management. Thomson, London.

[12] Degenne, A. and Lemel, Y. (2006) Sociologie des comportements intentionnels. Economica Editions.

[13] Wittorski, R. (1997) Analyse du travail et production des compétences collectives. L’Harmattan Ed.

[14] Cohen, M., Jaffray, J.-Y. and Said, T. (1987) Experimental Comparison of Individual Behavior under Risk and under Uncertainty for Gains and for Losses. Organizational Behavior and Human Decision Process, 39, 1-22. http://dx.doi.org/10.1016/0749-5978(87)90043-4

[15] Beattie, J. and Loomes, G. (1997) The Impact of Incentives upon Risky Choice Experiment. Journal of Risk and Uncertainty, 14, 155-168. http://dx.doi.org/10.1023/A:1007721327452 
Scientific Research Publishing (SCIRP) is one of the largest Open Access journal publishers. It is currently publishing more than 200 open access, online, peer-reviewed journals covering a wide range of academic disciplines. SCIRP serves the worldwide academic communities and contributes to the progress and application of science with its publication.

Other selected journals from SCIRP are listed as below. Submit your manuscript to us via either submit@scirp.org or Online Submission Portal.
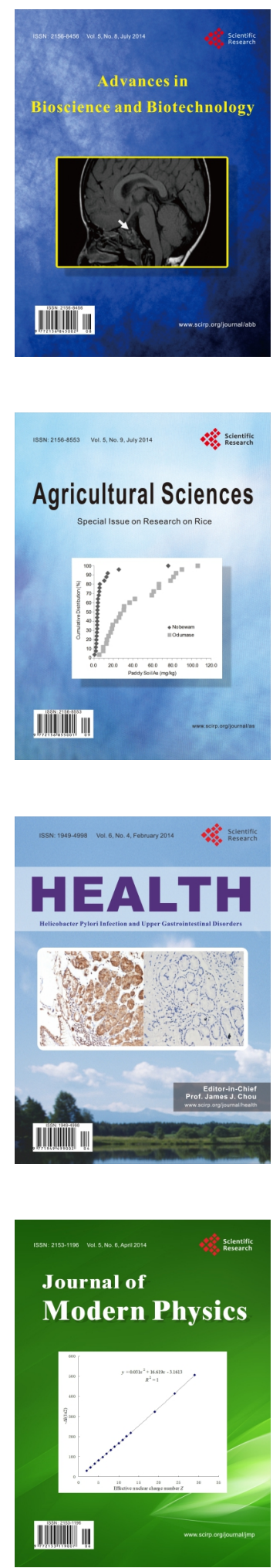
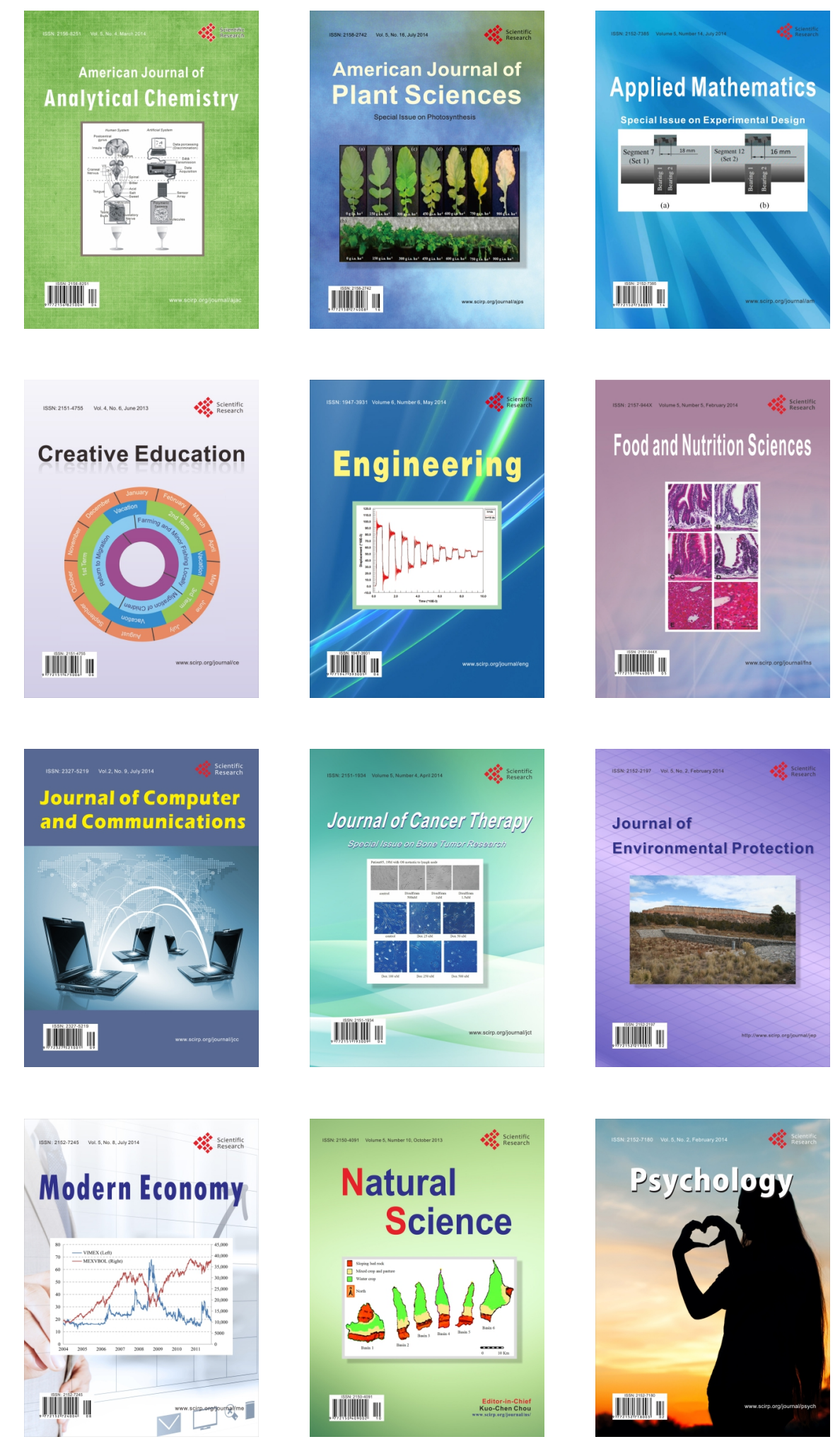